\title{
VALIDAÇÃO DO TEMPO DE VIDA ÚTIL APRESENTADO POR SENSOR FOTOCRÔMICO DE TEMPO E TEMPERATURA
}

ANA PAULA RESEM BRIZIO *

CARLOS PRENTICE **

\begin{abstract}
Estudou-se o comportamento de indicador fotocrômico de tempo e temperatura (ITT) com o objetivo de analisar a influencia de diferentes tempos de ativação (exposição à luz ultravioleta) sobre o processo de descoloração da etiqueta, caracterizando sua vida útil em condições específicas de temperatura. Os valores de desvanecimento da cor do ITT, quantificados mediante o chroma $b^{\star}$ (CIELab) em função do tempo, apontaram que a descoloração do rótulo é diretamente proporcional à quantidade de luz usada no processo de ativação e inversamente proporcional à temperatura de armazenamento da etiqueta. Devido à possibilidade de definir a vida útil do ITT de forma reprodutível concluiu-se que esse dispositivo constitui ferramenta confiável para monitorar a cadeia de frio de muitos produtos alimentares.
\end{abstract}

PALAVRAS-CHAVES: INDICADORES DE TEMPO ARMAZENAMENTO; TEMPOS DE ATIVAÇÃO; VIDA ÚTIL.

\section{E TEMPERATURA; TEMPERATURA DE} \begin{abstract}
Grande, RS (e-mail: anabrizio@yahoo.com.br).
** Professor Associado, FURG, Rio Grande, RS (e-mail dqmprent@furg.br).
\end{abstract}

* Doutoranda em Engenharia e Ciência de Alimentos, Universidade Federal do Rio Grande (FURG), Rio 


\section{INTRODUÇÃo}

A temperatura constituiu o fator mais importante que afeta a qualidade e a segurança dos alimentos durante sua produção, distribuição e armazenamento (TAOUKIS, 2008; TAUKIS, 2001). O desconhecimento do histórico de temperatura do alimento torna extremamente difícil prever sua vida útil (SHIMONI, ANDERSON e LABUZA, 2001). Assim, o ponto-chave do problema consiste em monitorar e controlar a temperatura da cadeia de frio desde a produção do alimento até o consumidor final (KREYENSCHMIDT et al., 2010). Nesse sentido, embalagens inteligentes contendo sensores de tempo e temperatura (ITT) poderiam satisfazer os requisitos acima descritos (VERDADE, 2010).

Define-se indicador ou integrador de tempo e temperatura (ITT) como dispositivo simples, pequeno e barato que pode mostrar mudanças facilmente mensuráveis, dependentes do tempo e da temperatura, e que refletem o histórico de temperatura do produto alimentar ao qual encontra-se anexado (TAOUKIS e LABUZA, 1989; TAOUKIS, LABUZA e FRANCIS, 1991; GIANNAKOUROU et al., 2005).

O OnVu ${ }^{\mathrm{TM}}$ ITT $\mathrm{B}_{1}$, indicador de tempo e temperatura irreversível, é utilizado para alimentos em condições de resfriamento $\left(0\right.$ a $\left.4{ }^{\circ} \mathrm{C}\right)$ e seu funcionamento baseia-se em reação fotocrômica em estado sólido. Essa reação ocorre devido à presença de cristais orgânicos que mudam de cor de acordo com as condições de tempo e temperatura. Tais cristais são a base do pigmento usado para formar a tinta inteligente do ITT, a qual muda de cor (de incolor para azul) após irradiação com luz ultravioleta (ativação). Depois de ativada, a tinta inteligente retorna ao estado incolor numa taxa dependente da temperatura (KREYENSCHMIDT et al., 2010; NGA, 2010), conforme ilustrado na Figura 1.

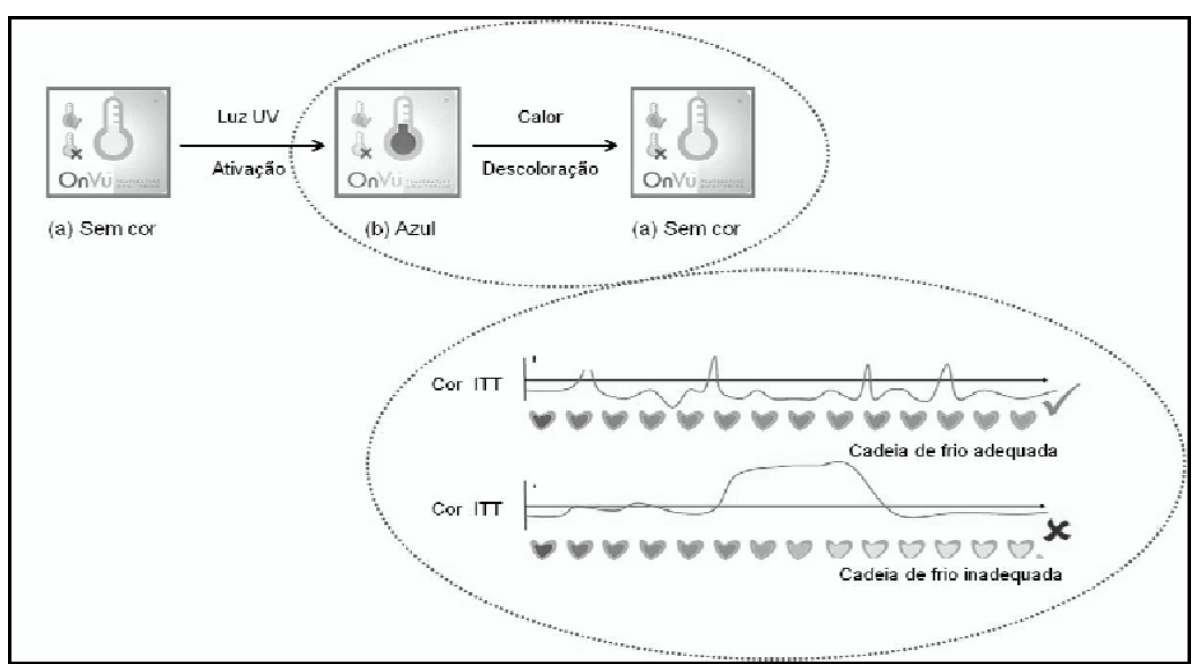

\section{FIGURA 1 - CICLO DE REAÇÃO DO SISTEMA FOTOCRÔMICO. A EXPOSIÇÃO À LUZ ULTRAVIOLETA (UV) INDUZ A COLORAÇÃO ENQUANTO O CALOR PROMOVE A REAÇÃO INVERSA}

A vida útil do ITT corresponde ao tempo que a cor da mancha fotocrômica azul da etiqueta ativada demora para alcançar a cor referência (NGA, 2010). A descoloração, proporcional à quantidade de luz empregada no processo de carga, pode ser ajustada pelo controle da duração e intensidade do pulso da luz ultravioleta (UV) utilizada na ativação do pigmento fotocrômico. O processo de desvanecimento da cor, e consequentemente o fim da vida útil do ITT, precisa ser calibrado tendo em conta principalmente a data de validade do alimento em que o indicador será anexado (KREYENSCHMIDT et al., 2010).

Este trabalho teve por objetivo determinar a vida útil da etiqueta inteligente OnVu ${ }^{T M}$ ITT $\mathrm{B}_{1}$, estudando distintas condições de energia de ativação de maneira a determinar a dependência entre o tempo de carregamento e o processo de descoloração da etiqueta, quando exposta a condições ideais $\left(0\right.$ a $\left.4{ }^{\circ} \mathrm{C}\right)$ e não ideais de temperatura de armazenamento $\left(>4{ }^{\circ} \mathrm{C}\right)$. 


\subsection{ONVU $^{\mathrm{TM}}$ INDICADOR DE TEMPO E TEMPERATURA}

Analisou-se o indicador de tempo e temperatura de impressão $O n V u^{T M}$ rótulo $\mathrm{B}_{1}$ (Ciba Specialty Chemicals e Freshpoint, Basel, Suíça, patente WO/2006/048412). No escuro, as etiquetas não carregadas são estáveis e não mudam de cor com o tempo durante períodos prolongados (12 meses), mesmo à temperatura ambiente.

Usou-se carregador de luz ultravioleta manual, desenvolvido para o OnVu ${ }^{T M}$ (GLP TTI, Bizerba, Alemanha) para ativar as etiquetas (Figura 2). Depois de carregados, os rótulos foram cobertos com filtro ótico sob a forma de fita de transferência térmica para protegê-los de recarregamento pela luz solar. A irradiação com luz UV da etiqueta foi medida em segundos (s).

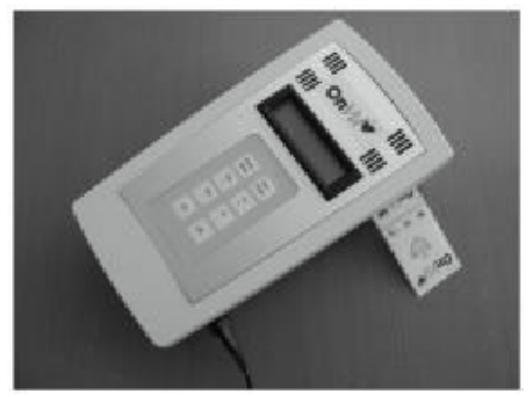

\section{FIGURA 2 - EQUIPAMENTO UTILIZADO PARA ATIVAR AS ITT}

\subsection{DELINEAMENTO EXPERIMENTAL}

Para analisar o efeito do tempo de ativação e a dependência da temperatura sobre o processo de descoloração das etiquetas, seis (6) diferentes tempos de carga e três (3) condições de armazenamento isotérmico foram investigados.

As temperaturas de armazenamento das etiquetas foram selecionadas considerando duas das mais importantes etapas de distribuição de produtos frescos: o ponto de venda e o subsequente armazenamento doméstico. Escolheu-se a temperatura de $3^{\circ} \mathrm{C}$ porque representa frequentemente a condição recomendada para o armazenamento de produtos refrigerados $\left(0\right.$ a $\left.4{ }^{\circ} \mathrm{C}\right)$, enquanto que as demais condições de estoque foram baseadas nos trabalhos realizados por Zhang et al. (2012), Limbo et al. (2010) e Cárdenas, Giannuzzi e Zaritzky (2008).

As etiquetas foram ativadas nas condições $1 ; 2 ; 3 ; 4 ; 5$ e 6 segundos (1 s de carga corresponde a $50 \mathrm{~mJ} / \mathrm{cm}^{2}$ de energia). Após a ativação, as etiquetas foram anexadas em placas de vidro pré-arrefecido e armazenadas em três incubadoras com temperatura de alta precisão (Marconi B.O.D MA 415/S, São Paulo, Brasil). Avaliaram-se as temperaturas de $3^{\circ} \mathrm{C}$ (condição ideal), $7^{\circ} \mathrm{C}$ e $10^{\circ} \mathrm{C}$ (condições de abuso) $\pm 0,5^{\circ} \mathrm{C}$, sendo monitoradas a cada 2 minutos por coletores de dados (Data Logger DHT5012, Perceptec, São Paulo, Brasil). O experimento foi conduzido duas vezes e cada tempo de ativação avaliado em triplicata. Controlou-se a temperatura ambiente durante o procedimento de ativação em $20 \pm 1,0^{\circ} \mathrm{C}$.

Mensurou-se o processo de descoloração das etiquetas diariamente com auxílio de colorímetro (Minolta Chroma Medidor de CR-210, Minolta, Osaka, Japão) através do sistema de cor CIELab (CIE, 1986). Obteve-se a dimensão do chroma b*, coordenada que quantifica a variação de cor de amarelo $\left(b^{\star}+\right)$ à azul $\left(b^{\star}-\right)$. As análises foram interrompidas quando $b^{\star}=0$ em que nenhuma mudança de cor azul adicional poderia ser medida na etiqueta.

O fim de vida útil do ITT foi definido como o tempo que a cor da mancha fotocrômica azul da etiqueta carregada levou para chegar até a cor referência. Essa cor final foi estabelecida mediante 
controle visual e posterior medição do chroma $b^{*}$, quando a cor do ITT apresentava tonalidade azul clara (quase cinza), conforme mostra a Figura 3.

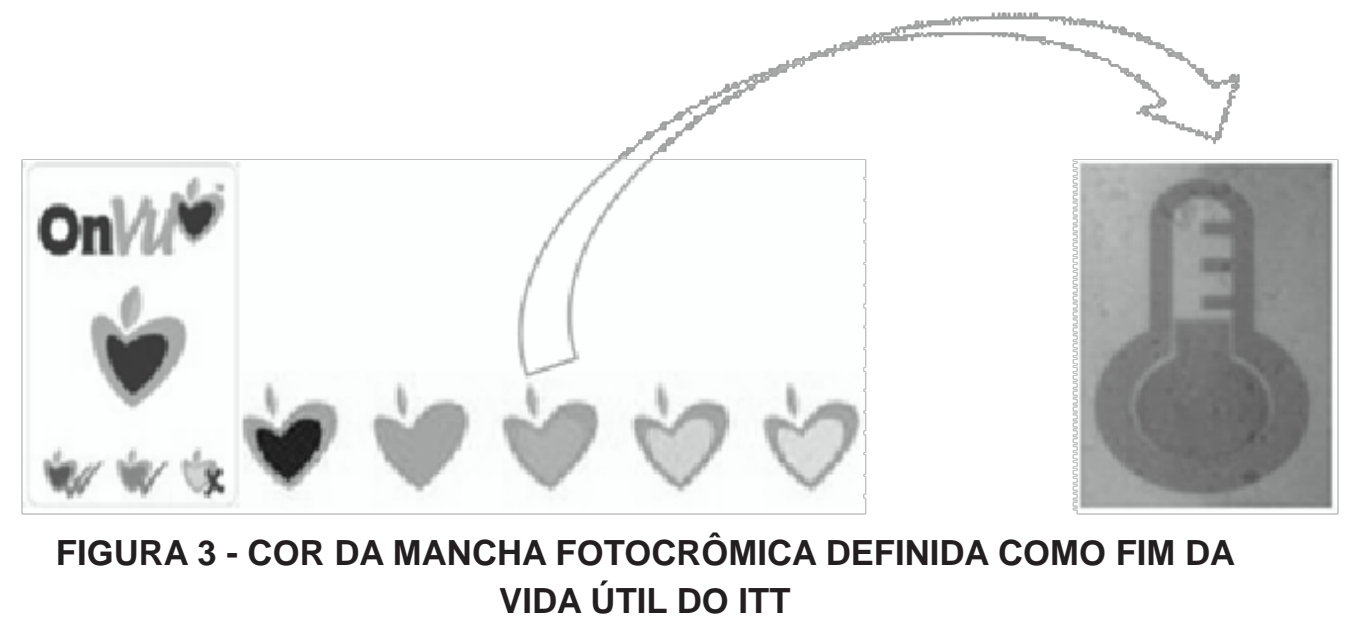

\subsection{REPRODUTIBILIDADE DO PROCESSO DE ATIVAÇÃO DOS ITT}

Avaliou-se a reprodutibilidade do processo de ativação usando o total de cinquenta e quatro (54) etiquetas e nove (9) ITT para cada tempo de ativação (1; 2; 3; 4; 5 e 6 s). Essas foram carregadas e imediatamente avaliadas em colorímetro pelo sistema de cor CIELab, obtendo-se a dimensão $b^{*}$.

\subsection{ANÁLISE DOS DADOS}

Utilizou-se o programa Microsoft Excel 2007 (Microsoft, Redmont, WA, EUA) para calcular médias, desvios padrão e construção dos gráficos.

Para avaliação da reprodutibilidade do processo de ativação dos ITT, os resultados dos valores de chroma $b^{*}$ mensurados nas etiquetas foram submetidos à Análise Descritiva Quantitativa, através do programa Statistica 7.0, utilizando a Análise de Variância (ANOVA) e o Teste de Tukey, com nível de significância de $5 \%(p<0,05)$ (NGA, 2010).

\section{RESULTADOS E DISCUSSÃO}

A confiabilidade do sistema de ITT constitui característica necessária e importante para a sua implementação na gestão da cadeia de frio (SHIMONI, ANDERSON e LABUZA, 2001). A etiqueta $O n V u^{T M}$ ITT exige processo de carga reprodutível porque variações do processo de ativação levam a tempos diferentes de duração.

A Tabela 1 mostra os valores médios obtidos para o parâmetro de cor chroma b*, durante a avaliação da reprodutibilidade do processo de ativação da etiqueta. O processo de carga mostrou variação não significativa $(p>0,05)$ para os tempos analisados, resultado que garante a confiabilidade do procedimento de carregamento. Cabe salientar que estudos realizados por Kreyenschmidt et al. (2010) e Verdade (2010) constataram que mudanças nas condições ambientais influenciam o processo de ativação das etiquetas. Segundo tais autores, temperaturas ambientais crescentes levam a períodos mais longos de descoloração em tempos de carga constante devido, principalmente, a maior transferência de energia para o rótulo. Portanto, a manutenção de condições de temperatura ambiental constante durante o carregamento das etiquetas constitui pré-requisito do processo de ativação dos ITT.

$\mathrm{Na}$ Tabela 1 pode ser observado que a intensidade da cor azul (valores de chroma $\mathrm{b}^{*}$ ) para as etiquetas ativadas durante $1 \mathrm{~s}\left(50 \mathrm{~mJ} / \mathrm{cm}^{2}\right.$ de energia) apresentaram valores significativamente inferiores aos medidos nas demais situações de carga $(p<0,05)$. 
TABELA 1 - IMAGEM E VALORES MÉDIOS DE CHROMA B* PARA AS ETIQUETAS CARREGADAS EM DIFERENTES TEMPOS DE ATIVAÇÃo

\begin{tabular}{|c|c|c|c|c|c|c|}
\hline & \multicolumn{6}{|c|}{ Tempo de ativação (s) } \\
\hline & 1 & 2 & 3 & 4 & 5 & 6 \\
\hline & $-28,97 \pm 0,30$ & $-28,97 \pm 0,30$ & $-29,82 \pm 0,36$ & $-29,29 \pm 0,28$ & $-29,64 \pm 0,55$ & $-29,14 \pm 0,31$ \\
\hline Chroma & & & & & & \\
\hline$b^{*}$ & $O_{n V u}$ & OnVu & OnVu & OnVu & OnVu & OnVu \\
\hline
\end{tabular}

A Figura 4 mostra o processo de descoloração do indicador quando mantido em temperatura ideal de conservação $\left(3 \pm 0,5^{\circ} \mathrm{C}\right)$. $\mathrm{O}$ tempo total do processo de desvanecimento da cor apresentou clara dependência do tempo de carregamento das etiquetas.

As amostras exibiram boa reprodutibilidade durante o processo de descoloração a $3 \pm 0,5^{\circ} \mathrm{C}$, apresentando baixos desvios (de zero a 0,97 ) entre os valores das triplicatas medidas para cada condição de carregamento. Resultados semelhantes foram encontrados por Kreyenschmidt et al. (2010), os quais avaliaram o processo de descoloração do OnVu ${ }^{T M}$ ITT $B_{1}$ com tempos de ativação entre 550-1600 ms.

Considerando chroma $b^{*}=-7,0$ como o valor que representa o último estágio da cor azul do ITT visualmente perceptível, as etiquetas mantidas em condições de temperatura ideais apresentaram duração de aproximadamente 3,5 (84 h); 6,5 (156 h); 9 (216 h); 11 (264 h); 12,5 (300 h) e $12(288$ h) dias para os tempos de ativação de $1 ; 2 ; 3 ; 4 ; 5$ e 6 segundos, respectivamente (Figura 4).

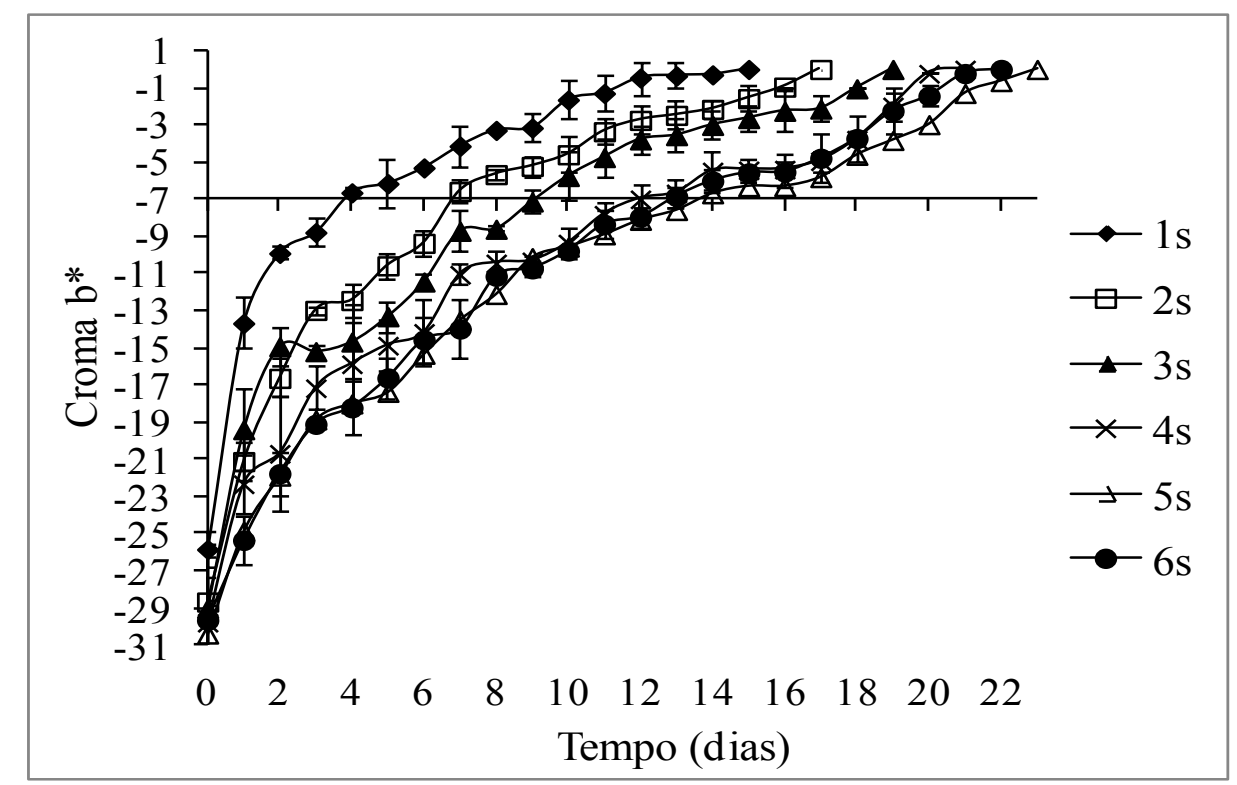

FIGURA 4 - DESCOLORAÇÃO DAS ETIQUETAS DURANTE ARMAZENAMENTO EM CONDIÇÃO IDEAL DE TEMPERATURA $\left(3^{\circ} \mathrm{C}\right)$

Nota: As barras de erro representam o desvio padrão das triplicatas de cada medição.

As Figuras 5 e 6 apresentam o processo de descoloração das etiquetas quando mantidas em temperaturas de 7 e $10 \pm 0,5^{\circ} \mathrm{C}$, respectivamente. 


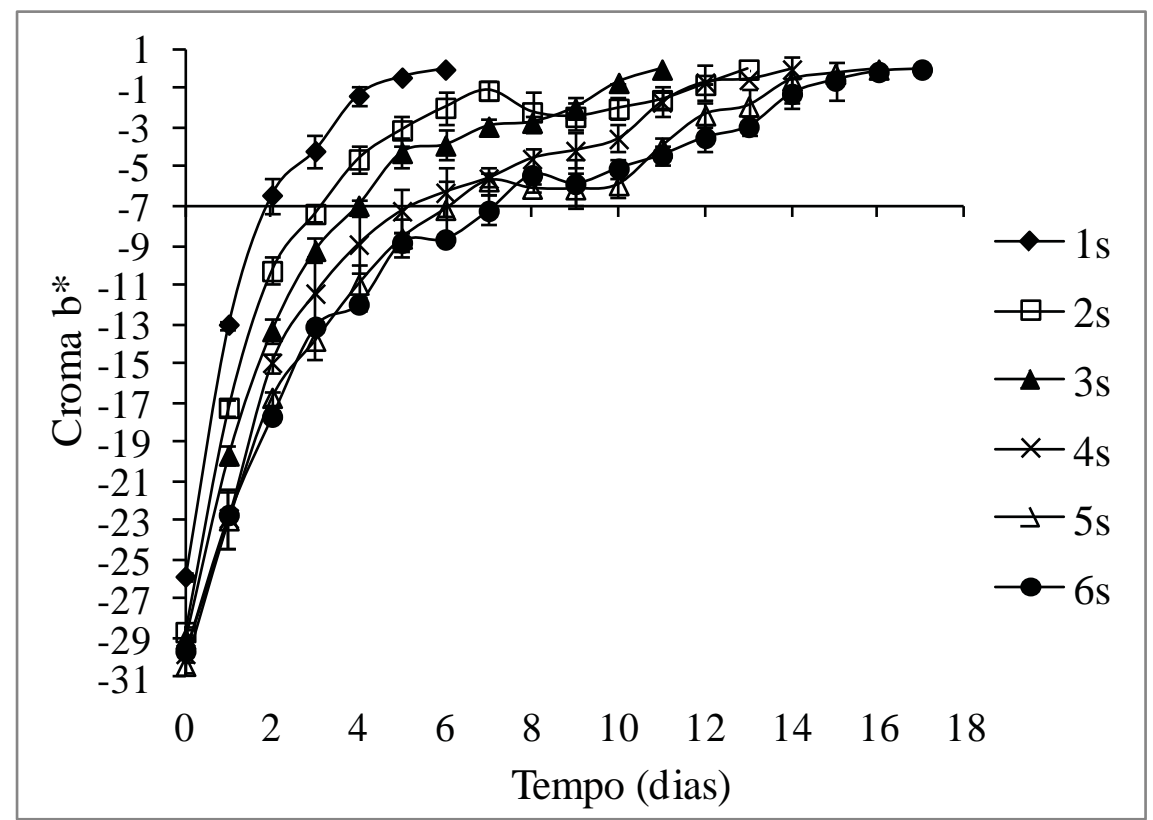

FIGURA 5 - DESCOLORAÇÃO DAS ETIQUETAS DURANTE ARMAZENAMENTO A $7^{\circ} \mathrm{C}$

Nota: As barras de erro representam o desvio padrão das triplicatas de cada medição.

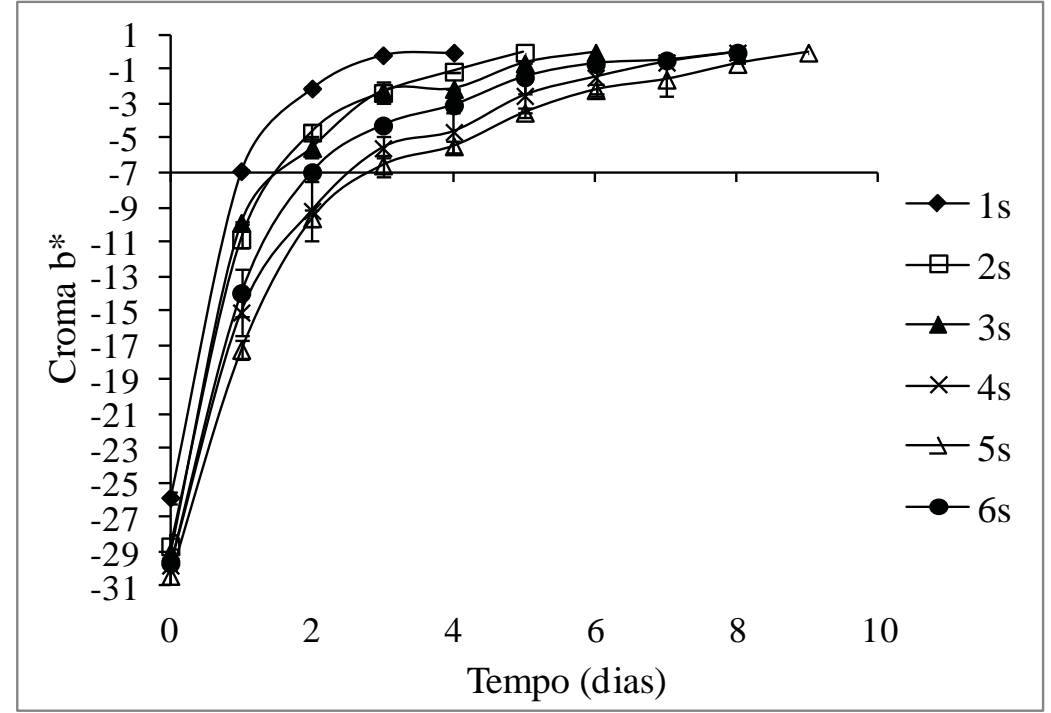

FIGURA 6 - DESCOLORAÇÃO DAS ETIQUETAS DURANTE ARMAZENAMENTO A $10^{\circ} \mathrm{C}$

Nota: As barras de erro representam o desvio padrão das triplicatas de cada medição.

O ITT mostrou boa reprodutibilidade do processo de descoloração sob condições de abuso de temperatura (desvio padrão entre zero e 0,98 ). A mudança de cor da etiqueta foi nitidamente acelerada durante o armazenamento em temperaturas superiores a ideal (máximo $4{ }^{\circ} \mathrm{C}$ ).

As etiquetas mantidas sob condições isotérmicas de $7 \pm 0,5^{\circ} \mathrm{C}$, considerando o fim da sua vida útil quando $b^{*}=-7,0$, apresentaram duração aproximada de 2 (48h); 3 (72 h); 4 (96 h); 5 (120 h); 6 (144 h) e 5,5 (132 h) dias de validade para 1; 2; 3; 4; 5 e 6 segundos de ativação, respectivamente 
(Figura 5). Já os ITT mantidos a $10 \pm 0,5^{\circ} \mathrm{C}$ apresentaram em torno de 1 (24 h); 1,5 (36 h); 2 (48 h); 2,5 (60 h); 3 (72 h) e 2 (48 h) dias de duração para as mesmas energias de ativação supracitadas, respectivamente (Figura 6 ).

As etiquetas expostas à luz UV durante $6 \mathrm{~s}$ pareceram exibir efeito de supersaturação (TSIRONI et al., 2011) e sua resposta atingiu valores mais baixos do que o esperado. Assim, recomenda-se o uso do intervalo de carregamento entre 1 a 5 segundos.

Devido à possibilidade de determinar o período de vida útil do ITT usando diferentes tempos de ativação, a etiqueta pode ser adaptada de maneira muito flexível à validade do alimento. Deste modo, o indicador pode ser usado em diferentes produtos desde que sua taxa de desvanecimento seja calibrada com a taxa de deterioração do alimento.

\section{CONCLUSÃO}

Os resultados do estudo mostraram que o processo de desvanecimento da cor azul do rótulo em função do tempo apresenta relação diretamente proporcional à quantidade de luz usada no processo de ativação e inversamente proporcional a temperatura de armazenamento da etiqueta. Devido à possibilidade de definir a vida útil do $O n V u^{T M} I T T B_{1}$ de forma reprodutível esse dispositivo constitui ferramenta confiável para monitorar as cadeias de refrigeração de produtos alimentares.

\section{ABSTRACT}

\section{VALIDATION OF SHELF-LIFE TIME PRESENTED BY A PHOTOCHROMIC TIME-TEMPERATURE INDICATOR}

In the present paper the behavior of a photochromic time-temperature indicator (TTI) was studied in order to analyze the influence of different activation times on the process of label discoloration, featuring its shelf-life in specific temperature conditions. The values of color fading of the TTI quantified by chrome $b^{*}$ (CIELab) as a function of time, showed that the label discoloration is directly proportional to the amount of light used for the activation process and inversely proportional to the temperature of the label storage. Due to its capacity of determining in a reproducible way the shelf-life of TTI, this was considered a reliable tool to monitor the cold chain for many food products.

KEY-WORDS: TIME-TEMPERATURE INDICATORS; STORAGE TEMPERATURE; ACTIVATION TIMES; SHELF-LIFE.

\section{REFERÊNCIAS}

1 CÁRDENAS, F.C.; GIANNUZZI, L.; ZARITZKY, N.E. Mathematical modelling of microbial growth in ground beef from Argentina. Effect of lactic acid addition, temperature and packaging film. Meat Science, v. 79, p. 509-520, 2008.

2 Commission International de l'Éclairage (CIE). Colorimetry. Wien, Austria, 1986. p.88-107. (Publication CIE, 15.2).

3 GIANNAKOUROU, M.C.; KOUTSOUMAINS, K.; NYCHAS, G.J.E.; TAOUKIS, P.S. Field evaluation of the application of time temperature integrators for monitoring fish quality in the chill chain. International Journal of Food Microbiology, v.102, p.323-336, 2005

4 KREYENSCHMIDT, J.; CHRISTIANSEN, H.A.; HUBNER, V.R.; PETERSEN, B. A novel photochromic time-temperature indicator to support cold chain management. International Journal of Food Science and Technology, v.45, n.2, p.208215, 2010.

5 LIMBO, S.; TORI, L.; SINELLI, N.; FRANZETTI, L.; CASIRAGHI, E. Evaluation and predictive modeling of shelf life of minced beef stored in high-oxygen modified atmosphere packaging at different temperatures. Meat Science, v.84, p.129136, 2010.

6 NGA, M.T.T. Enhancing quality management of fresh fish supply chains through improved logistics. 2010. $226 \mathrm{~s}$. Thesis (PhD in Food Science) - Faculty of Food Science and Nutrition (School of Health Sciences), University of Iceland, Reykjavik, 2010.

7 SHIMONI, E.; ANDERSON E.M.; LABUZA T.P. Reliability of time temperature indicators under temperature abuse. Journal of Food Science, v.66, n.9, p.1337-1340, 2001. 
8 TAOUKIS, P.S. Application of time-temperature integrators for monitoring and management of perishable product quality in the cold chain. In: KERRY, J.P. Smart packaging technologies for fast moving consumer goods. Chichester: John Wiley \& Sons, 2008. p. 61-74.

9 TAOUKIS, P.S. Modelling the use of time-temperature indicators in distribution and stock rotation. In: FOOD process modelling. Cambridge: Woodhead Publishing, 2003. p. 402-428.

10 TAOUKIS, P.S.; LABUZA, T.P.; FRANCIS R.C. Time-temperature indicators as food quality monitors. Food Packaging Technology, v.1113, p.51-63, 1991.

11 TAOUKIS, P.S.; LABUZA, T.P. Applicability of time-temperature indicators as shelf life monitors of food products. Journal of Food Science, v.54, p.783-788, 1989.

12 TSIRONI, T.; STAMATIOU, A.; GIANNOGLOU, M.; VELLIOU, E.; TAOUKIS, P.S. Predictive modelling and selection of time temperature integrators for monitoring the shelf life of modified atmosphere packed gilthead seabream fillets. Food Science and Technology, v.44, p.1156-1163, 2011

13 VERDADE, P.S. Estudo de um TTI fotocrómico para aplicação em alimentos refrigerados. 2010. 120 f. Dissertação (Mestrado em Bioquímica e Química dos Alimentos) - Universidade de Aveiro, Portugal, 2010.

14 ZHANG, Q.Q.; HAN, Y.Q.; CAO, J.X.; XU, X.L.; ZHOU, G.H.; ZHANG, W.Y. The spoilage of air-packaged broiler meat during storage at normal and fluctuating storage temperatures. Poultry Science, v.91, p.208-214, 2012.

\section{Agradecimentos}

Ao CNPq pelo apoio financeiro e pelas bolsas concedidas. À empresa Basf pelo apoio e material fornecido para a execução deste trabalho. 